\title{
Approaches and limitations to the prediction of energy requirements in wild animal husbandry and veterinary care
}

\author{
BY JAMES K. KIRKWOOD AND PETER M. BENNETT \\ Veterinary Science Research Group, Institute of Zoology, Zoological Society of London, Regent's
} Park, London NW1 4RY

There are circumstances when estimates of food or energy requirements are an important component of the successful husbandry and veterinary care of wild animals (Kirkwood, 1991). However, for most of the 20000 species of terrestrial vertebrates, these requirements have not been measured at any stage of their life-cycles. It is, therefore, necessary to make predictions based on findings in other species.

There is a large literature which examines variation in rates of energy expenditure, particularly at basal or near to basal level, within and between species. Much of this is concerned with functional or adaptive explanations for the observed variation and its allometric relationship with body mass. However, allometric equations summarizing interspecies variation also offer a means for making predictions and have been used for this in a variety of contexts.

In the present paper, the issue of how energy requirements, particularly for maintenance in captivity, can be predicted for individuals of one species on the basis of patterns of variation between other species will be addressed and some of the caveats and limitations in this process will be examined. Although the subject of the present paper is energy requirements, the points addressed are of general relevance in the use of allometric equations for prediction.

\section{APPROACHES TO ESTIMATING ENERGY REQUIREMENTS IN THE ABSENCE OF SPECIES-SPECIFIC DATA}

Methods for estimating the metabolizable energy (ME) requirement $(\mathrm{kJ} / \mathrm{d})$ of an individual wild animal for existence in captivity will be examined. This quantity, the existence metabolic rate $(\mathrm{EMR} ; \mathrm{kJ} / \mathrm{d})$, is that required to sustain energy balance in captive conditions and it includes limited activity and thermoregulatory costs.

The estimation of food intake necessary to meet the ME requirement is relatively straightforward. The metabolizability of the gross energy of a food (the proportion available for metabolism) is dependent on the nature of the food and the digestive efficiency of the animal eating it. There is quite an extensive literature on the metabolizability (or efficiency of assimilation) of the gross energy of foods consumed by a wide range of species. For example, Robbins (1983) provided a useful table of metabolizability values.

EMR can be estimated from an allometric equation relating EMR to body mass, if a suitable equation is available, or as a multiple of measured or predicted basal metabolic rate (BMR; $\mathrm{kJ} / \mathrm{d}$ ). BMR, the rate of heat production when resting and fasting (or, in some cases, under more strictly defined basal conditions) has been measured in more than 365 species of birds (Bennett \& Harvey, 1987) and at least 685 species of mammals (Heusner, 1990). These values correspond to about $5 \%$ of bird species and about $17 \%$ of mammalian species. Measurements of EMR have been made in far fewer species. 
Before addressing the problems of using allometric equations for predictions, some consideration will be given to the issue of estimating EMR from BMR.

\section{ESTIMATION OF EMR AS A MULTIPLE OF MEASURED OR PREDICTED BMR}

If data are available on the BMR then an estimate of the EMR can be derived. Many authorities suggest that, in the absence of better information, EMR can be estimated as a multiple of BMR (or an estimate of it). For example, it is often suggested that EMR may be about two times (e.g. Robbins, 1983; Ullrey \& Allen, 1986; Blaxter, 1989), or 1.5 times BMR (e.g. Clarke et al. 1977) and a comparison of some regressions is shown in Table 1. It is not possible to give a precise value because the relationship is not a precise one. The activity, thermoregulation and other components of EMR vary, and the efficiency of utilization of metabolizable energy depends on the chemical nature of the food and the digestive physiology of the species eating it. It has been found to vary, for example, from 0.98 in single-stomach animals fed on fat to 0.7 in ruminants fed on protein (Blaxter, 1971, 1989).

\section{PREDICTING EMR OR BMR FROM ALLOMETRIC EQUATIONS}

Allometric equations can be used for predicting EMR or BMR from body mass. However, it is important to consider the quality of the equation used for this purpose and how accurate the estimate generated by it is likely to be.

Several authors have drawn attention to the methodological problems of exploring between-species variation in the relationship between BMR and mass. These difficulties (see below) have to be borne in mind when attempting to interpret the significance of relationships between BMR and body mass or when using these allometric relationships as a basis for comparisons between taxa (Harvey \& Mace, 1982; Calder, 1984; Hayssen \& Lacy, 1985; Bennett, 1986; Bennett \& Harvey, 1987). The limitations are also relevant to the use of these relationships for predictive purposes. These points and some others relevant to using allometric equations for predictive purposes will be listed and discussed.

\section{METHODOLOGICAL PROBLEMS OF MAKING PREDICTIONS FROM ALLOMETRIC EQUATIONS}

(a) The regression method. Allometric equations are dependent on the statistical method used in their derivation (Harvey \& Mace, 1982). Unless the correlation of $X$ and $Y$ is high, different values may be obtained for the slopes and intercepts depending on whether linear regression, major axis or reduced major axis is computed. An assumption in linear regression is that $X$ is measured without error. When, as is the case with metabolic rate and body mass, the measurement of both variables is subject to error, linear regression may give a biased estimate of the slope. Alternatively, when both $X$ and $Y$ are subject to error, the slope of the major axis or reduced major axis can be calculated. The major axis is the principal (longest) axis of an ellipse describing the bivariate distribution and the reduced major axis is that derived after transforming the variables such that the means and standard deviations of each are zero and one (Sokal \& Rohlf, 1986). 
Table 1. Comparison of allometric equations relating basal metabolic rate (BMR) and existence metabolic rate (EMR) ( $\mathrm{kJ} / \mathrm{d})$ to body mass $(\mathrm{W} ; \mathrm{kg})$ in some taxa*

\begin{tabular}{lrlrc} 
& $\begin{array}{c}\text { BMR } \\
(\mathrm{kJ} / \mathrm{d})\end{array}$ & & $\begin{array}{c}\text { EMR } \\
(\mathrm{kJ} / \mathrm{d})\end{array}$ & EMR:BMR \\
\hline Birds & $333 \mathrm{~W}^{0 \cdot 67}$ & Accipitridae & $460 \mathrm{~W}^{0.67}$ & $1 \cdot 4$ \\
Mammals & $293 \mathrm{~W}^{0.75}$ & Mammals & $611 \mathrm{~W}^{0.75}$ & $2 \cdot 1$ \\
Reptiles & $28 \mathrm{~W}^{0.77}$ & Snakes & $45 \mathrm{~W}^{0.75}$ & $1 \cdot 6 \dagger$ \\
Primates & $242 \mathrm{~W}^{0.78}$ & Primates & $405 \mathrm{~W}^{0.75}$ & $1 \cdot 7 \dagger$ \\
\hline
\end{tabular}

* From Kleiber, 1961; Evans \& Miller, 1968; Bennett \& Dawson, 1976; Kirkwood, 1981; Kirkwood \& Underwood, 1984; Bennett \& Harvey, 1987; Ross, 1989; J. K. Kirkwood and C. Gili, unpublished results.

$\dagger$ Approximate values.

(b) The taxonomic level of analysis. The nature of the relationship between metabolic rate and body-weight is dependent on the taxonomic level at which the analysis is carried out (Harvey \& Mace, 1982, Heusner, 1982; Hayssen \& Lacy, 1985; Bennett \& Harvey, 1987). An analysis carried out between species (taking mean BMR and mass for each) within a family may yield a different result than a between-species within-class analysis (Fig. 1). Likewise the result obtained in an analysis of BMR and mass between species within a family may be different from the result of analysis between families within a class. Bennett \& Harvey (1987) demonstrated variation in the allometric exponent in their analysis of resting metabolic rate in relation to mass in birds, from greater than 0.9 between species within genera, to less than 0.7 between sub-orders within class.

(c) Unequal representation of taxa. Biases may be introduced by unequal representation of taxa. This could occur if, for example, several data points are taken for one species but only one each for others (Fig. 2), or if the sample contains many representatives from one family but none or few from others (Harvey \& Mace, 1982; Bennett, 1986; Bennett \& Harvey, 1987).

(d) Taxonomic accuracy. Allometric equations for given taxa (e.g. families or orders) are dependent on the correctness of the classification system on which they are based (Fig. 3). There are many cases where classification remains in dispute: for example, as to
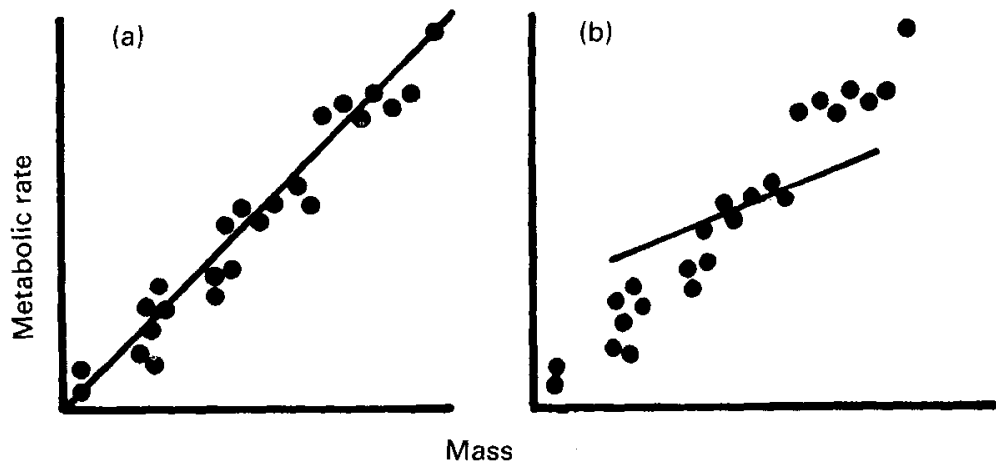

Fig. 1. Relationship between metabolic rate and body mass can be influenced by the taxonomic level of the analysis. Each point represents a species. The regression for a data set across wide taxonomic grouping, e.g. a class or an order, can differ from that of a sub-set, e.g. a family or genus, within it. 


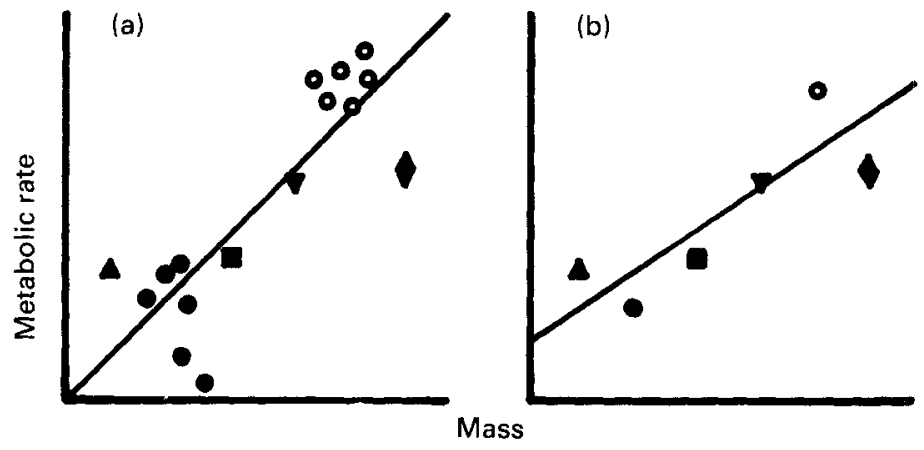

Fig. 2. Relationship between metabolic rate and body mass can be influenced by unequal representation of taxa. (a) Shows how unequal representation of taxa, e.g. more than one point per species or more than one species per family, may bias the regression. (b) Six taxa are equally represented (each once).
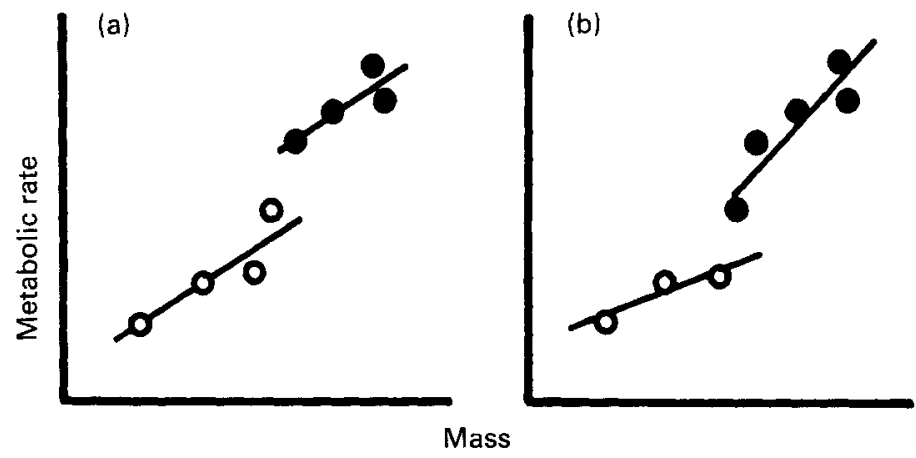

Fig. 3. Relationship between metabolic rate and body mass are dependent on the reliability of the classification system. (a), (b) Illustrate how the metabolic rate $v$. body mass relationships of two groups (e.g. families within orders) may depend on taxonomic assumptions. Here the regressions depend on whether the central point is grouped with $(\mathcal{O})$ or $(\bigcirc)$.

whether certain taxa are species or sub-species, and as to the order to which some families belong (Bennett, 1986).

(e) Data quality. The quality of data available on metabolic rates of wild animals is variable and, since some workers tend to concentrate on particular taxa (e.g. rodents, ungulates), there is the potential for bias due to variation in methodology.

(f) Body mass range. When regressions are calculated using data from animals spanning only a narrow range in body mass, there is great potential for errors in measurement of the parameter which varies with mass, to seriously bias the estimates of slope or intercept (Blaxter, 1989). It follows that predictions based on body masses that fall outside the range used in calculating the regression are particularly hazardous.

(g) The precision of predictions from allometric equations. It is necessary, when attempting to make predictions from allometric equations (or regressions), to have an understanding of the likely precision of the prediction. The standard error of a predicted value of $Y$ (SE $Y^{\prime}$ ) can be calculated from the following equation (Sokal \& Rohlf, 1981): 
SE $Y^{\prime}=s^{2} y \cdot x\left(1+\frac{1}{n}+\frac{(X i-\bar{X})^{2}}{\Sigma x^{2}}\right)$,

where $s^{2} y . x$ is the root mean square of the regression, $n$ is the sample size, $X i$ is the value of $X$ for which a $Y$ estimate is required, $\bar{X}$ is the mean value of $X$ in the sample, and $\Sigma x^{2}$ is the sum of squares of $X$. The upper and lower $95 \%$ prediction limits ( $L 1$ and $L 2)$ for the estimate of $Y$ at $X i$ can be calculated:

$$
L 1=Y^{\prime}+t_{0.05(n-2)} \cdot \operatorname{SE} Y^{\prime},
$$

and, $L 2=Y^{\prime}-t_{0.05(n-2)} \cdot \mathrm{SE} Y^{\prime}$,

where the value $t_{0.05(n-2)}$ is taken from a table of the Student's $t$ distribution for $P=0 \cdot 05$ and df $n-2$.

These limits predict the range within which the likelihood that a $Y$ value for a given $X$ will fall is $95 \%$. They are wider than the $95 \%$ confidence limits of the regression because there is greater uncertainty about predicting the likely position of an individual point than of the regression. It is important to note that even when correlation coefficients are high, the upper and lower $95 \%$ limits of a prediction often span a wide range. The limits are narrowest at the $X$ value corresponding to the mean weight of the sample from which the regression is derived. At these points the upper and lower $95 \%$ prediction limits of the few regressions examined were about twice $(1 \cdot 4-2 \cdot 0)$ and half $(0 \cdot 43-0 \cdot 7)$ the predicted $y$ values respectively (Table 2 ).

Another problem is that the upper and lower $95 \%$ prediction limits cannot be calculated from the statistics usually provided in the literature, and there is no simple relationship with the correlation coefficient that enables their estimation. Prediction limits have to be calculated from the regression statistics derived from the raw data. Allometric equations, such as those in Table 2, describing the relationship between metabolic rate and body mass are quite abundant in the literature but the information required for assessing their predictive precision is rarely available.

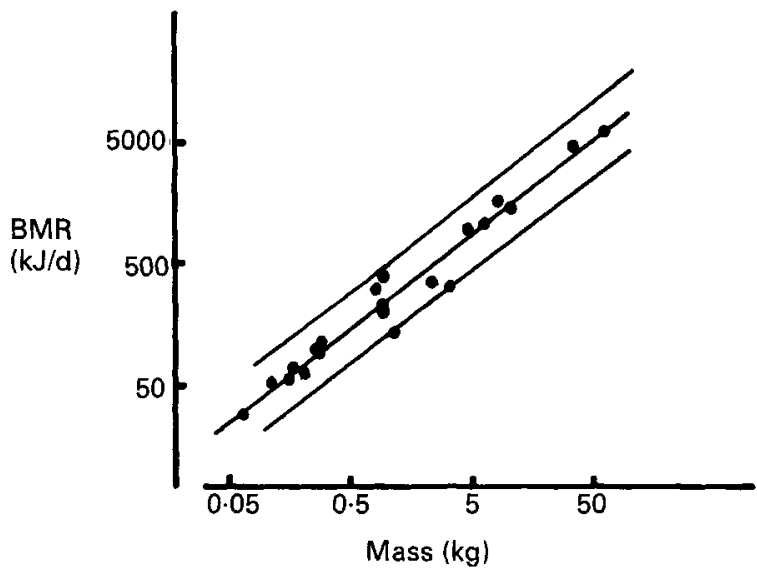

Fig. 4. Even when there is a close correlation between basal metabolic rate (BMR) and body mass, the $95 \%$ prediction limits of the regression can be wide. This figure shows a linear regression for BMR $v$. body mass for twenty-one species of primates and the $95 \%$ prediction limits of the regression $(r 0.979, n 21)$. The data for this regression were taken from Ross (1989), some points are species means, others are measurements on individual animals. 


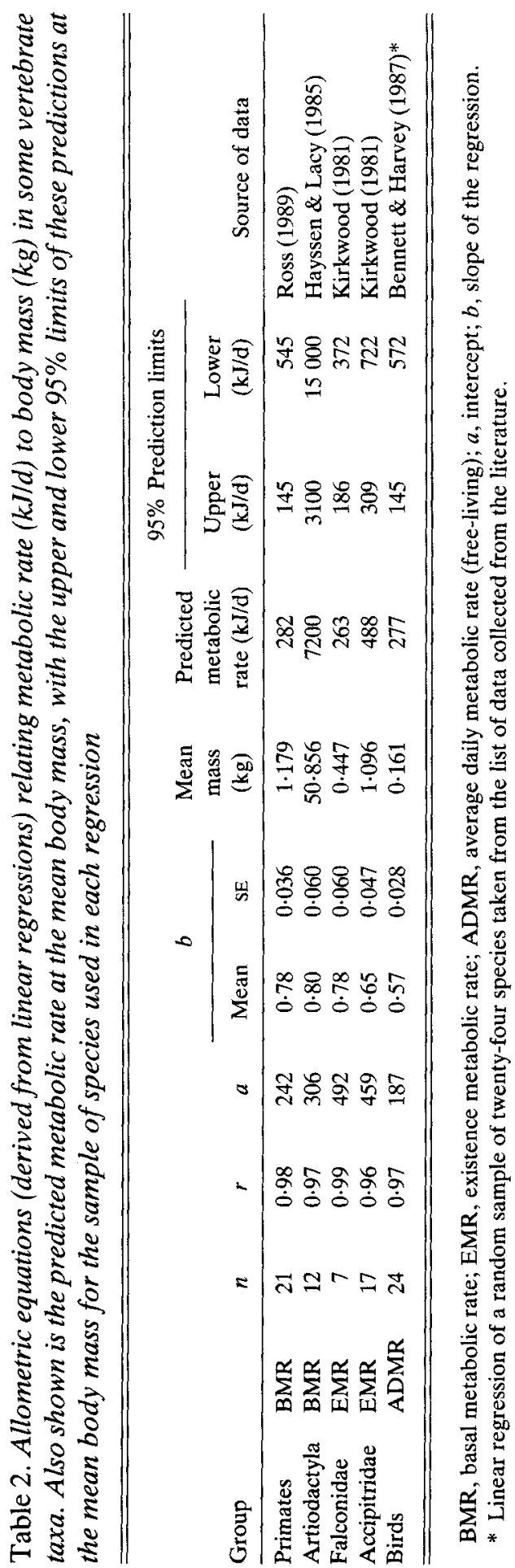


(h) Equations based on species means predict species means not individual requirements. When the regressions used in making predictions have been derived using species means for metabolic rate and mass (as is usually the case), the values predicted are species means and not predictions for individual animals. This limitation cannot be avoided.

\section{DISCUSSION AND CONCLUSIONS}

Due to lack of specific information it is often necessary, in the course of wild animal husbandry and veterinary care, to predict the energy and food requirements of individuals. Metabolic rate is partly dependent on body mass and allometric equations describing this relationship offer a means of making such predictions. However, there are a number of caveats and limitations that should be observed.

Allometric equations are dependent on the methods used in their derivation and on the sample of taxa involved. There is no uniquely correct method for determining the nature of the relationship between metabolic rate and mass in the animal kingdom. Major axis or linear regression can be used depending on the circumstances, and the choice of the taxonomic level at which the analysis is carried out depends on the hypothesis to be tested (Bennett \& Harvey, 1987). Ideally, to predict a parameter (for example, the metabolic rate of an animal from its body-weight), a regression based on a sample of similar animals (i.e. same species, sex and age) should be used. When data are not available for similar animals the next best approach is to predict the mean for the species using a regression based on a sample of species. Regressions based on analyses at higher taxonomic levels, such as between families within a class, may provide useful guidelines but obviously predict means for those higher taxonomic levels rather than species means.

The regression chosen for making the prediction will depend upon the quality of those available. Broad species-within-a-class regressions such as that of Kleiber (1961) or of Hayssen \& Lacy (1985) may be less accurate than those based on a sample of species more closely related to the species for which the prediction is required. If regressions of species within orders or within families are available these may be more useful for predictive purposes, but their quality will be influenced by the sample size and the weight range they span.

When making predictions about the requirements of an individual animal from allometric equations, often the best that can be done is to estimate the species mean using the best-fit line estimated by the appropriate equation and statistical technique. However, it is important to recognize that even when correlations are high, the limits within which there is a $95 \%$ chance that the species mean will fall are often wide, and that the requirements of specific individuals will vary about this mean.

\section{REFERENCES}

Bennett, A. F. \& Dawson, W. R. (1976). Metabolism. In Biology of the Reptilia, vol. 13, pp. 127-223 [C. Gans and F. H. Pugh, editors]. New York: Academic Press.

Bennett, P. M. (1986). Comparative studies of morphology, life history and ecology among birds. PhD Thesis, University of Sussex.

Bennett, P. M. \& Harvey, P. H. (1987). Active and resting metabolism in birds: allometry, physiology and ecology. Journal of Zoology 213, 327-363. 
Blaxter, K. L. (1971). Methods of measuring the energy metabolism of farm animals and interpretation of the results obtained. Federation Proceedings 30, 1436-1443.

Blaxter, K. L. (1989). Energy Metabolism in Animals and Man. Cambridge: Cambridge University Press.

Calder, W. A. (1984). Size, Function and Life History. Cambridge, Mass., USA: Harvard University Press.

Clarke, H. E., Coates, M. E., Eva, J. K., Ford, D. J., Milner, C. K., O'Donoghue, P. N., Scott, P. P. \& Ward, R. J. (1977). Dietary standards for laboratory animals: report of the Laboratory Animals Centre Diets Advisory Committee. Laboratory Animals 11, 1-28.

Evans, E. \& Miller, D. S. (1968). Comparative nutrition, growth and longevity. Proceedings of the Nutrition Society 27, 121-129.

Harvey, P. H. \& Mace, G. M. (1982). Comparisons between taxa and adaptive trends: problems of methodology. In Current Problems in Sociobiology, pp. 343-361 [King's College Sociobiology Group, editors]. Cambridge: Cambridge University Press.

Hayssen, V. \& Lacy, R. C. (1985). Basal metabolic rates in mammals: taxonomic differences in the allometry of BMR and body mass. Comparative Biochemistry and Physiology 81A, 741-754.

Heusner, A. A. (1982). Energy metabolism and body size. 1. Is the 0.75 mass exponent of Kleiber's equation a statistical artifact? Respiration Physiology 48, 1-12.

Heusner, A. A. (1990). Basal metabolism and body mass in dogs. Abstracts of the Wallham International Symposium on Nutrition of Small Companion Animals, p. 8. Davis, Calif.: University of California.

Kirkwood, J. K. (1981). Maintenance energy requirements and rate of weight loss during starvation in birds of prey. In Recent Advances in the Study of Raptor Diseases, pp. 153-157 [J. E. Cooper and A. G. Greenwood, editors]. Keighley: Chiron Press.

Kirkwood. J. K. (1991). Energy requirements for maintenance and growth of wild mammals, birds and reptiles in captivity. Journal of Nutrition 121, S29-S34.

Kirkwood, J. K. \& Underwood, S. J. (1984). Energy requirements of captive cotton-top tamarins (Saguinus oedipus oedipus). Folia Primatologica 42, 180-187.

Kleiber, M. (1961). The Fire of Life. New York: John Wiley \& Sons.

Robbins, C. T. (1983). Wildlife Feeding and Nutrition. New York: Academic Press.

Ross, C. A. (1989). Life history strategies of primates. PhD Thesis, University College London.

Sokal, R. R. \& Rohlf, F. J. (1986). Biometry. New York: W. H. Freeman \& Co.

Ullrey, D. \& Allen, M. E. (1986). Principles of zoo animal nutrition. In Zoo and Wild Animal Medicine, pp. 516-532 [M. E. Fowler, editor]. Philadelphia: W. B. Saunders Co. 